\title{
Addressing Strategic Knowledge Gaps in the Search for Biosignatures on Mars
}

A White Paper Submitted to the Planetary Science and Astrobiology Decadal Survey 2023-2032

\author{
Lead Author \\ Nathalie A. Cabrol, \\ SETI Institute Carl Sagan Center \\ 189 Bernardo Avenue, Suite 200, Mountain View, CA 94043 \\ ncabrol@seti.org(650)8100226
}

\begin{abstract}
Coauthors
Janice Bishop, SETI Institute, jbishop@seti.org, Sherry L. Cady, Pacific Northwest National Laboratory, sherry.cady@pnnl.gov, Cecilia Demergasso, Universidad Catolica del Norte, Antofagasta, cdemerga@ucn.cl, Nancy Hinman, University of Montana, nancy.hinman@mso.umt.edu, Michael Hoffman, University of Montana, michael.hofmann@umontana.edu, Isik Kanik, Jet Propulsion Lab, isik.kanik@jpl.nasa.gov, Jeffrey Moersch, University of Tennessee, Knoxville, jmoersch@,utk.edu, Nora Noffke, Old Dominion University, NNoffke@odu.edu, Victor Parro, Centro de Astrobiología (CSIC-INTA), parrogv@cab.inta-csic.es, Cynthia Phillips, Jet Propulsion Lab, cynthia.b.phillips@jpl.nasa.gov, Michael Phillips, University of Tennessee, Knoxville, mphill58@,vols.utk.edu, Laura Sánchez Garcia, Centro de Astrobiología (CSIC-INTA), 1sanchez@cab.inta-csic.es, Pablo Sobron, SETI Institute, psobron@seti.org, David Summers, SETI Institute, dsummer@seti.org, Kimberley Warren-Rhodes, SETI Institute, krhodes@seti.org. David Wettergreen, Carnegie Mellon University, Pittsburgh,dsw@cmu.edu, Miguel Angel Férnandez-Martínez, McGill University, miguel.fernandezmartinez@mcgill.ca, Virginia Gulick, SETI Institute, vgulick@seti.org
\end{abstract}

See the list of $\mathbf{3 7}$ cosigners here:

https://www.dropbox.com/scl/fi/n5xi5uy26hjed0b0pn96w/Book.xlsx?cloud_editor=excel\&dl=0 \&rlkey=p91psyprsre5dxygeg4oubbzp

All authors and cosigners have given permission to be named on this document 


\section{Overview: The Current Challenge}

With the search for biosignatures, the exploration of Mars is shifting from the characterization of habitability to that of the coevolution of the planet, i.e., the spatiotemporal interactions life may have had with its environment. At present, the intellectual framework underpinning the preparation of Mars 2020 and ExoMars along with future life-seeking missions is, however, essentially the same as the one that has guided the exploration of Mars for the past 15 years [1-4]. This framework is articulated around the terrestrial analogy principle of habitability. While this principle has been helpful in characterizing Mars' habitability potential over time (and that of any planet), it is limiting - and potentially misleading - for the exploration of biosignatures as it focuses primarily on the spatiotemporal dynamics and general geographic distribution of environmental factors. To advance our understanding of the distribution of life on Mars, the search must shift from habitable environments to evidence for coevolution. This new context will facilitate the intellectual and technological advancements required to search for extinct and extant inhabited environments more efficiently and holistically.

Coevolution synergistically considers planetary history, and both life and environment. As a result, it is a more effective, systemic, and dynamic approach than habitability alone for understanding how to detect, identify, and characterize (past/present) microbial habitats and biosignatures. New paths of investigation must be developed to advance our understanding of plausible coevolution models on early Mars, and to support biosignature exploration. They include (1) revisiting intellectual frameworks, theories, hypotheses, and science questions from a coevolutionary perspective; (2) injecting an ecosystem view at all levels of biosignature exploration [5] i.e., spatiotemporal scales, spectral resolution, orbit-to-ground detection and identification thresholds [e.g., 6-7], landing site selection, and exploration strategies; (3) designing and deploying new mission concepts to gain a high-resolution view of environmental variability at scales that are relevant to (past/present) Martian microbial habitats, and (4) developing novel technologies and instruments capable of interrogating microbially-relevant scales and distinguishing between biotic and abiotic patterns, or their interactions.

\section{Coevolution as a Guiding Exploration Principle}

Biological processes on Mars would have taken place within the distinct context of an irreversible early collapse of the magnetosphere and atmosphere [e.g., 8], greater climate variability and gradients, and specific geographic, planetary and astronomical characteristics. These comprise the unique coevolutionary constraints that would have separated a Martian biosphere from that of Earth very early in its history. To evaluate their full effect on all possible biosignatures, these constraints should be envisioned within an intellectual framework that includes life as an interactive agent of transformation of its environment, and a piece of a dynamic system of polyextreme environmental conditions with complex loops and feedback mechanisms.

\subsection{Intellectual Framework}

The concept of habitability - defined by the range of the environments (astronomical, planetary) within which life as we know it could survive - drives current astrobiology exploration strategies. Life is regarded as a passive actor in an environment that provided (or failed to provide) water, energy, nutrients, and shelter for prebiotic and biological processes. In this context, the concept of habitability does not imply life; it simply considers environmental conditions for its emergence and sustainability.

The habitability and preservation potential of early Mars has now been demonstrated by more than 20 years of orbital and landed missions [9-12], as has the presence of organic molecules [13- 
17]. The upcoming missions will test the hypothesis that life has developed on Mars and left evidence of its presence [18]. Testing this hypothesis holistically requires a search for the traces left by two dynamic agents (life and environment) that modified each other as cause or effect [19]. As Earth shows, coevolution affects physicochemical, geochemical, and biological processes at all scales, including e.g., biological architecture, metabolic activity, morphology, the mineralogy and texture of soils and sediments, topography, the atmosphere, microbial habitats, biological dispersal, biomass production and repositories, and biosignature preservation. It is, therefore, a concept essential to biosignature exploration. A coevolutionary approach to biosignature exploration allows core hypotheses and science questions to be reframed on the basis of plausible spatiotemporal synergies between life and environment, and to infer relevant spatial scales and spectral resolution. Examples include:

Hypothesis A: Prebiotic and biological processes as we know them developed on early Mars.

Example Questions: (1) What role did environmental differences between Earth and Mars play in an early evolution of life on Mars? (2) What was the impact of unique physical features (e.g., global dichotomy, high obliquity, lost magnetosphere and atmosphere, volcanic and tectonic characteristics) on the formation and spatiotemporal evolution of environmental pathways for biological dispersal, and biomass/biosignature repositories? (3) What does a comparison between the timing of early life evolution on Earth and the current environmental models for early Mars suggest about ancient habitable environments, habitat development potential, biological dispersal, biosignature preservation, detection threshold? (4) Models of early Mars are inferred from what we understand of early Earth, but to what extent do we see biological influences on surface environments in our geologic record of the early Earth, i.e., aren't we pinning biological stamps on the preserved environmental record, and is there a way to better understand early primary production? (5) What does the lack of obvious biosignatures at current resolution suggest about (a) the extent and duration of subaerial habitats, biomass accumulation and preservation potential, and (b) the detection and identification thresholds of integrated instrument payloads required from orbit to the ground?

\section{Hypothesis B: Mars developed a second, independent, and distinct genesis.}

Example Questions: (1) What distinct biological traits (e.g., metabolism, morphology, behavior, structure, size, biogeochemical cycles) could have evolved from the unique terms of a Martian coevolution (astronomical, planetary, environmental, geographic, climatic, tectonism and sedimentary cycles, other), and (2) what distinct traces of coevolution could they have left in the geological or spectral records? For instance, how can existing datasets be searched for unique geochemical, mineralogical, morphological, textural, and biochemical markers that could stem from life's adaptation to the Martian polyextreme environment?

\section{Hypothesis C: Life never developed on Mars - No coevolution.}

Example Questions: What are the critical exploratory steps to complete at the surface, subsurface, and deep underground, (and where), before such a conclusion can be reached? And how can we combine and mine the datasets from all Mars missions efficiently while new missions are readied?

\subsection{Understanding Coevolution in a Polyextreme Environment}

A Martian coevolution would have been imprinted early by the development of a polyextreme environment $[5,20]$. While the current approach to biosignature exploration considers multiple extreme factors, it often analyzes their impact individually, with limited attempts at a systemic and holistic approach, i.e., without characterizing microbial-environmental interactions and their lasting effects, [21-23]. Terrestrial analogs of such environments demonstrate that interactions 
between multiple extreme environmental factors (e.g., UV radiation, thin atmosphere, aridity) generate complex loops and feedback mechanisms at various scales through combinations that may alternatively either magnify, decrease, and/or cancel their individual effects, and often override global (planetary) trends at the microbial-habitat scale [5, 19]. Further, the mitigation of any one of these extreme factors triggers a new dynamic that life has to adapt to, as shown by the humidity-salinity feedback [24].

- Key 1. Understanding the spatiotemporal interplay of polyextreme factors, the resulting interactions with biological processes, and the resulting biogeosignatures is key to conceptualizing a Martian coevolution and finding bona fide biosignatures.

At global to regional scales, the unique complexity of Mars - including during its early geological history - resided in the relative dominance of these polyextreme factors over space and time. Some parameters declined with time (magnetosphere, atmosphere, energy), while others had distinct spatiotemporal effects that depended upon obliquity (e.g., water, ice distribution). For example, while the loss of the atmosphere was ultimately linked to the loss of the magnetic field, weak fields play a lesser role in surface radiation doses than the loss of the atmospheric depth [23]. Changes in atmospheric shielding were therefore not only a factor of time, but also a consequence of obliquity [21-22], and this unpredictability in the radiation environment was only one of many variables (e.g., changes in temperature, desiccation, geochemistry and sediment texture, acidity) that life had to contend with. At regional to local scale, microenvironments created by a combination of geomorphic and geologic processes (e.g., impact, volcanic, or magmatically generated hydrothermal systems) combined with other factors such as low elevations resulting in high surface pressures may have permitted unique and persistent habitats throughout Mars' history [25-26].

- Key 2. Understanding how this variability affected prebiotic and biological processes, as well as the development and footprint of microbial habitats, is critical for evaluating plausible biomass production, potential biosignature formation and preservation, and appropriate detection levels for instruments.

At local (habitat) scales, the footprint and sustainability of microbial habitats in terrestrial analogs of extreme environments depend on microclimates generated by synergies between microbial metabolic activity and local environmental factors, that trigger unique loops and feedback mechanisms. Changing environmental conditions would have thus affected habitats in a systemic way, with modifications and/or loss in connectivity networks, formation and isolation of microniches, and the production of very localized and specific sets of ecosystem conditions.

- Key 3. Modeling plausible metabolic pathways and responses to variable polyextreme environmental factors is key for understanding adaption and survival potential of subaerial habitats over time, their spatiotemporal distribution, and biosignature formation and preservation potential.

While a diversity of biosignatures can be searched for, proteins and lipids have been shown to preserve critical information on both microbial metabolisms and their ability to biomediate their mineralogical environment. These molecules are preserved in polyextreme environments, allowing inference of past metabolic pathways [27, 28].

On Earth, polyextreme environments are the exception, that is, they are regional extreme departures from a generally more mesic surface, which receive biological subsidies from the mesic Earth, including their biota (generally consisting of extremely tolerant organisms). 
- Key 4. Considering that Mars became polyextreme early in its history, (i.e. mesic early Mars is polyextreme Earth), it is critical to understand whether polyextreme environments can be cradles for life, and what primary production, biomass, biosignature formation and biosignature preservation potential these conditions enable. This understanding is a fundamental step in recognizing what types of organisms may have survived, and where/how to search for them. Fieldwork and modeling supporting those goals should be prioritized.

\section{Strategic Research Goals \&Mission Support}

Assuming coevolution occurred on Mars, the current approach to landing site selection provides limited (contextual) support for biosignature exploration. Data at relevant spatial scales and spectral resolution are only available at the three rover landing sites and, unless a future mission returns to one of them, knowledge acquired at these sites may only be partially transferable to the exploration of a new site - i.e. only if sets of environmental conditions are repeated at a habitatrelevant scale, e.g., sediment maturity (composition and texture), mineralogy, geochemistry, texture, structure, insolation, slope, moisture, or other. Current knowledge gaps will not be filled by the time Mars 2020, ExoMars, and Tianwen launch. However, significant advances can be made, and support provided to upcoming and future missions through data analysis, theoretical modeling, lab experiments, fieldwork, High End Computing (HEC), Artificial Intelligence (AI), and machine learning (see also Fig. 1), including:

2.1 Loops and Feedback Mechanisms in Polyextreme Environments: Mars' ability to preserve subaerial habitats, ecotones, connectivity networks, and microbial dispersal pathways and biosignatures over time would have depended on fluctuating interactions between multiple environmental extremes and their relative dominance at any given time [5]. This relative dominance would have impacted the interactions between life and environment and the spatiotemporal nature (distribution, preservation, type, biochemistry, geochemistry, mineralogy, other) of biosignatures. Relative dominance must be thus characterized over geological timescales and with changing obliquities, including along depth (temporal) and lateral (spatial) gradients. This can be done through:

- Key 5. Lab experiments and fieldwork in extreme environments that combine multiple extreme factors relevant to Mars, emphasizing the characterization of their interactions and their effects on prebiotic, biological processes, microbial habitats, and microbial spatiotemporal distributions and preservation should be prioritized. Fieldwork in extreme environments should take into consideration geologic and environmental baseline conditions that are not transferable to Mars (e.g., sedimentary cycling, tectonics, others) but might influence loops and feedback mechanisms.

- Key 6. Libraries of biogeosignatures resulting from these interactions (e.g., spectral, morphologic, metabolic, genomic) should be generated at integrated scales from orbit to ground to lab. Libraries of characteristics and patterns distinguishing abiotic, biotic, and mutual interaction patterns should be developed.

- Key 7. Biosignature formation should be characterized through the lens of polyextreme environmental factors and their role on local scale microclimates, characteristics of microbial habitats (e.g. geology, morphology, mineralogy, sediment texture, structure, composition).

- Key 8. Biosignature preservation potential and redistribution has to be viewed through the lens of polyextreme environmental factors and conditions, from a regional (e.g., global climate patterns, tectonic, sediment cycling, other) to local (e.g., basin morphology, microclimate, provenance) to microenvironment (e.g., substrate composition, mineralogy, texture) scale. 
- Key 9. HEC-based theoretical modeling using datasets from past and present missions should support the quantitative and qualitative characterization of the spatiotemporal evolution of polyextreme interactions on Mars, including through episodic changes in obliquity. Characterization should include present-day Mars.

2.2 Coevolution, Biological Architecture, and Biosignatures: Crossing the uncertainty threshold (i.e. biosignature potential vs. confirmed biosignature) requires (a) the development of knowledge about how coevolution could have shaped a Martian biological architecture (e.g.,

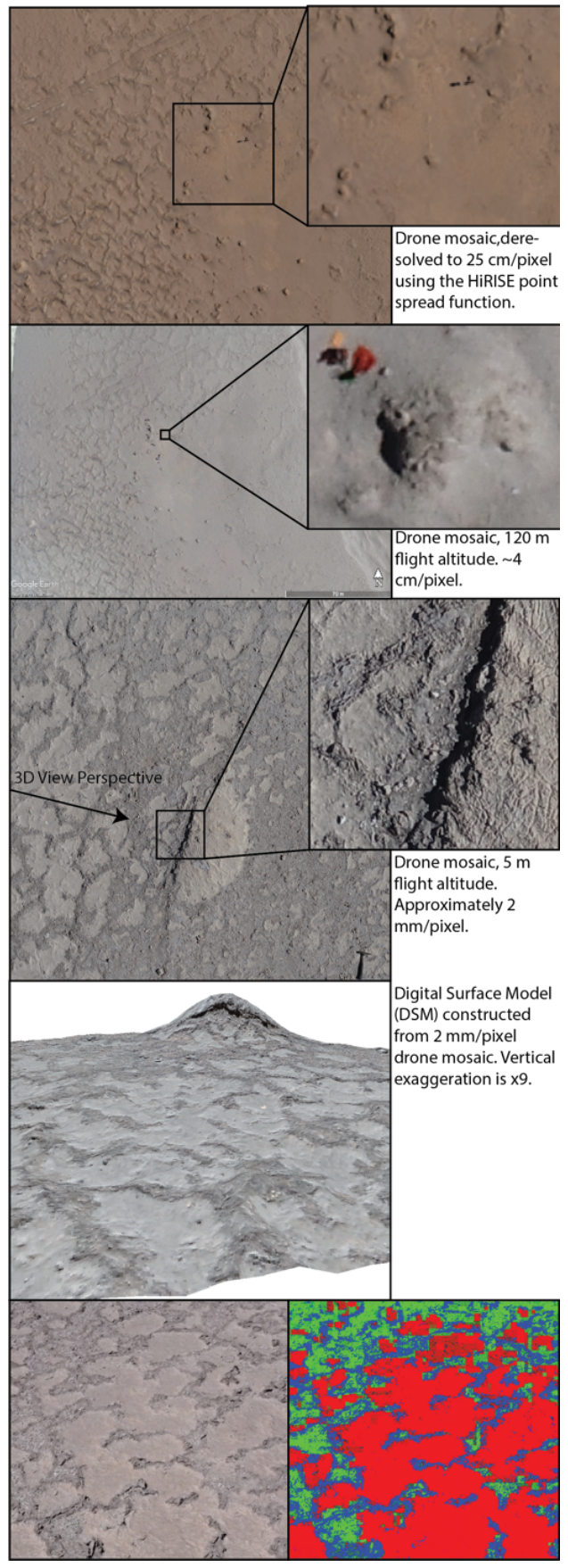
chemical structure, morphology, size, genetic makeup, metabolism) and its interactions with, and response to, a polyextreme environment; (b) prioritization of observations, and (c) an understanding of when a suite of observations constitutes an unambiguous and definitive confirmation of the presence of life. Filling the current knowledge gaps (e.g., origin and nature of life, biological architecture, biosignatures) demands the analysis of vast amounts of data from many scientific domains and envisions countless probabilistic occurrences. This is an area where Artificial Intelligence (AI) and machine learning can provide critical support for standard lab, field, and theoretical approaches and significantly speed up breakthrough discoveries. HEC-based theoretical modeling can provide the systemic environmental envelope to test scenarios for an origin of life and the spatiotemporal evolution of habitats.

Fig. 1. (From top to bottom): Ground to orbit data integration bridging orbital scale to habitat microscale using drone imagery, DEM and neural net classifiers for the identification of habitats. Data from the NAI SETI Institute Team, Salar de Pajonales, Chile.

- Key 10. Coevolution models for life as we know it can be generated by exploring datasets relative to prebiotic and biotic processes known from early Earth, which can be run through the environmental models. AI and machine learning can help accelerate the identification of unique (bio-geo)signatures across past and present mission data (orbital, landed, ground-based, and space observations) and foster the discovery of patterns of interactions from biological processes unique to Mars (life as we do not know it) with the environment.

\subsection{Ecosystem Approach to Landing Site Selection and}

Surface Operations: A coevolutionary approach calls for novel integrated investigation methods and techniques at specific spatial scales, spectral resolution, and detection/identification thresholds relevant to

(past/present) microbial ecosystems. Support includes: 
- Key 11. Engage microbiologists, biogeochemists, geneticists, ecologists, specialists in environmental sciences, extreme environments, and AI specialists early and at all stages: Programmatic, missions (concept and instrument payload design, science teams), and surface operations (exploration templates) to date include few of these specialists and do not readily facilitate their participation in mission design and execution.

- Key 12. Develop an integrated suite of missions and instruments that allow the identification of biogeosignatures from orbit to the ground. This requires a quantum leap in instrument capabilities and the development of novel analytical tools [29-32]. This is critical because mission simulations in extreme environments show that orbital resolution is of limited utility for Mars-relevant biosignature detection, and because finding evidence of potentially limited and heterogeneously distributed biomass may prove difficult from the ground alone [5].

- Key 13. Integrate survey techniques and spatiotemporal pattern analyses developed in microbial ecology into surface operation templates during missions [33-35] and convert datasets to a common language format for cross-correlation and integration to optimize mission findings gathered worldwide.

\section{Bridging Mission Concept}

While our understanding of early Mars environmental evolution still has key knowledge gaps [33-34], it is clear that Mars today is a reflection of the past three-and-half billion years. A characterization of the high-resolution scale of variability of the environment today has yet to be undertaken. Such a mission, completed with our knowledge of the role of climate forcing and obliquities, would reduce the risk and provide a low-uncertainty insight into the ecological potential of Mars's surface and near surface over 75\% of its history [5]. While life is not expected on the surface today, understanding present conditions, the influence of the landscape at scales and resolution that matter for microbial habitats on Mars (e.g., topography, geology, texture, albedo, mineralogy, other), will help to:

- Key 14. Identify surface expressions of seasonal and perennial microenvironment "hotspots" and show what types of microniches could have developed on the last habitable surface oases (e.g., To know what to search for). These data could be transferred back into models and AI.

- Key 16. Identify the environmental criteria/factors and scales to investigate (i.e., where and how to search, including: slope exposure, cracks in rocks, cavities in sediments, surfacelatmosphere interactions, temperature, moisture, light, mineralogy, sediment texture, $p H$, other), and how these search parameters would vary for extant versus extinct microbial life and its preserved biosignatures.

- Key 16. Conduct laboratory experiments simulating the Martian conditions to gain insights into both the degradation processes experienced by organic matter on Mars as well as possible preservation mechanisms through interaction with the minerals in the environment. This contributes to validate hypotheses about the nature of organic compounds detected so far on Mars and provides fundamental support to several aspects of life detection missions, such as: (i) establishing habitability of different Martian geological environments and the preservation potential of biomarkers; (ii) selecting landing sites and the most relevant samples to analyze in situ or to bring back to Earth in sample return missions; (iii) defining more accurately the molecular target for life detection missions depending on the product of transformation of possible biomarkers; (iv) developing life detection techniques and payload instruments suitable to detect and accurately identify specific compounds/features resulting from the alteration/preservation of biomarkers; (v) validating the potentialities of flight instruments 
through comparison with laboratory analogues; (vi) helping interpretation of data collected in situ or obtained through remote sensing.

Ultimately, this approach could become a cornerstone strategy for the exploration of Mars, as it supports three critical exploration goals: (1) the search for biosignatures, (2) human exploration (climate, weather, and activity planning) and (3) planetary protection, in that it may point to unexpected modern near-surface niches for life, and give more focus to the discussion about the potential conflicts between the search for biosignatures (where and how, see also Cabrol et al., Mission Concepts White Paper) and the impending human exploration [35].

\section{Beyond Mars}

These areas and related SKGs identify promising key research areas, science questions, and technology challenges in the field of planetary exploration and astrobiology. While they are presented here in the context of the exploration of Mars, coevolution, along with the questions, hypotheses, and approaches suggested here, could be regarded as primary guiding principles for the search for life in our Solar System and beyond.

\section{References:}

1. Mustard et al., 2012, Report of the Mars 2020 Science Definition Team, (MEPAG), 155-205; 2. Hayes et al., 2017, Astrobiology 17363-400; 3. Williford et al., 2018, In: From Habitability to Life, (Cabrol, N.A., and E.A. Grin, Eds), Elsevier, 275-309; 4. Vago et al., 2017, ESA Bull. 126:1623; ․ Cabrol, 2018, Astrobiology 18(1), doi: 1089/ast.2017.1756; ㅁ. Cabrol et al., 2017, AbSciCon, Mesa, Arizona. Abstract \#3033; 7. Phillips et al., 2017, Astrobiol. Sci. Conf., \#3373; $\underline{8}$. Jakosky et al., 2017, Science 355:1408-1410; . Knoll et al., 2005, Earth Planet Sci Lett 240:179-189; 10. Des Marais, 2010, Proc Am Philos Soc 154:402-21; 11. Summons et al., 2011 Astrobiology 11:157-181; 12. Grotzinger et al., 2015, Science 350, doi:10.1126/science.aac7575; 13. Chastain and Chevrier, 2007, Planet Space Sci 55:1246-1256; 14. Mumma et al., 2009, Science 323:10411045; 15. Blamey et al., 2015, Nat Commun 6, doi:10.1038/ncomms8399; 16. Freissinet et al., 2015, J Geophys Res: Planets 120:495-514; 17. Webster et al., 2015, Science 347 (6220): 415417; 18. Ruff and Farmer, 2016, Nat Commun 7, doi:10.1038/ncomms13554; 19. Knoll, 2009, Rend Lincei Sci Fis Nat 20:301-306; 20. Cabrol et al., 2007, Proc SPIE 6694. doi:10.1117/12.731506; 21. Jakosky and Phillips, 2001, Nature 412:237-244; 22. Kreslavsky and Head, 2005, Geophys Res Lett 32, doi:10.1029/2005GL02264;23. Atri et al., 2013, Astrobiology 13:910-919; 24. Azua-Bustos, A. et al. 2018. Sci Rep 8, 16706. https://doi.org/10.1038/s41598018-35051; 25 Gulick 1998, J. Geophys. Res. 103, 19365-19387; 26 Haberle et al. 2001, J. Geophys. Res: Planets 106, 23317-23326. 27. Sánchez-García, L. et al. 2020. Astrobiology, doi: 10.1089/ast.2019.2101; 28 Fernández-Martínez, M.A. et al., 2019. Front. Microbiol. doi: 10.3389/fmicb.2019.01641; 29. Thompson et al., 2011, J. Field Robotics. 28:542-564; 30. Tao and Muller, 2016, Planet Space Sci 121:104-113; 31. Candela et al., 2017, IEEE/RSJ Int. Conf. Intel. Robots and Syst. Vancouver, Canada; 32. Cabrol et al., 2007, J Geophys Res: Biogeosciences 112, doi:10.1029/2006JG000298; 33. Warren-Rhodes et al., 2007, J Geophys Res: Biogeo.112, doi:10.1029/2006JG000283; 34. Hock et al., 2007, J Geophys Res: Biogeosciences 112, doi:10.1029/2006JG000321; $\underline{35}$. Ehlmann et al., 2016, J Geophys Res: Planets 121, doi:10.1002/2016JE005134; 즈. Wordsworth 2016, Annu Rev Earth Planet Sci 44, doi:10.1146/annurev-earth-060115-012355; 37. Fairén, A.G., et al. 2017. Astrobiology, https://doi.org/10.1089/ast.2017.1703 